\title{
Le prologue des Géorgiques : une théorie du dessin?
}

\section{Martine Créac'H}

\section{(2) OpenEdition}

1 Journals

Édition électronique

URL : http://journals.openedition.org/ccs/900

DOI : $10.4000 /$ ccs. 900

ISSN : 2558-782X

Éditeur :

Presses universitaires de Rennes, Association des lecteurs de Claude Simon

\section{Édition imprimée}

Date de publication : 31 mai 2014

Pagination : 75-84

ISBN : 9782753533387

ISSN : $1774-9425$

\section{Référence électronique}

Martine Créac'H, «Le prologue des Géorgiques : une théorie du dessin ? », Cahiers Claude Simon [En ligne], 9 | 2014, mis en ligne le 22 septembre 2017, consulté le 01 mai 2019. URL : http:// journals.openedition.org/ccs/900; DOI : 10.4000/ccs.900 


\title{
LE PROLOGUE DES GÉORGIQUES: UNE THÉORIE DU DESSIN?
}

\author{
Martine CRÉAC'H \\ Université Paris 8 Vincennes-Saint-Denis
}

Sur la description du dessin à la mine qui précède les cinq grandes parties des Géorgiques, de nombreuses lectures ont déjà été proposées. Elles mettent en valeur "le statut réflexif " de ce texte où "s'énoncent indirectement les principes de composition de l'œuvre ${ }^{1}$ ". " Mise en abyme ", " préambule » ou «prologue », le texte est généralement considéré comme un modèle du récit à venir dont il anticipe, par ses moyens propres, la signification.

La scène est la suivante: dans une pièce de vastes dimensions un personnage est assis devant un bureau, l'une de ses jambes à demi-repliée sous son siège, le talon du pied soulevé, le pied droit en avant et plat, le tibia formant avec la cuisse horizontale un angle d'environ quarante-cinq degrés, les deux bras appuyés sur le rebord du bureau, les deux mains tenant au-dessus une feuille de papier (une lettre?) sur laquelle les yeux sont fixés. Le personnage est nu. [...] Un second personnage plus jeune, nu lui aussi, se tient debout de l'autre côté du bureau, dans la pose classique de l'athlète au repos, le poids du corps portant sur la jambe gauche, le bras droit pendant le long du corps, le bras gauche serrant contre la poitrine un carton rectangulaire sur lequel la main vient se $\operatorname{refermer}^{2}(G, \mathrm{p} .11-12)$

Cette proposition cependant mérite d'être interrogée. D’abord parce qu'elle présuppose que le trait commun entre le dessin et le récit est celui d'une histoire qui met entre parenthèses le traitement de celle-ci, autrement dit parce qu'elle met au second plan la facture du dessin. Or si, pour Claude Simon, les arts plastiques sont un modèle pour l'écriture, si le romancier « tra-

1. Brigitte Ferrato-Combe, Écrire en peintre. Claude Simon et la peinture, Ellug, université Stendhal, Grenoble, 1998, p. 95

2. Claude Simon, Les Géorgiques, Paris, Minuit, 1981, p. 11-17. Sauf indication plus précise, les citations appartiennent à ce passage. Le titre du roman Les Géorgiques sera abrégé en $G$. 
vaille comme un peintre ", c'est sans doute parce que le médium joue un rôle décisif pour lui.

Ensuite, parce que la lecture de la description comme préambule ou prologue est une lecture rétrospective, intellectuelle et savante qui suppose que la compréhension de l'enjeu de la description n'est accessible qu'au lecteur qui a déjà lu tout le livre. Or, si nous lisons cette description en oubliant provisoirement le récit qui va suivre, en nous abstenant d'y reconnaître ce que nous allons découvrir par la suite, nous sommes frappés par le caractère dépréciatif des jugements sur le dessin décrit et tentés d'en faire, plutôt qu'un modèle de l'écriture de Claude Simon, un contre-modèle.

C'est la lecture paradoxale que je voudrais proposer ici: Claude Simon, à l'orée de son roman, nous indiquerait non pas un "mode d'emploi pour la lecture " comme on l'a suggéré ${ }^{3}$, mais un contre-modèle de l'écriture de son roman.

Je voudrais, pour commencer, revenir sur les termes utilisés pour désigner ces quelques pages parce qu'ils en orientent l'interprétation en les plaçant sous la dépendance du texte qui va suivre. Pour étudier cette description, dont on sait qu'elle ne fut pas rédigée avant le roman, mais " plus tard, en cours de travail 4 ", je la détacherai ensuite du texte qu'elle précède, pour la comparer à d'autres descriptions de pratiques graphiques. Je reviendrai enfin aux Géorgiques pour tenter de mettre en relation une théorie du dessin et une théorie de l'écriture.

\section{FAÇONS DE DIRE}

Préambule, mise en abyme, prologue: ces trois termes ne sont pas, bien sûr, purement descriptifs. Ils nous invitent à une réflexion critique sur le rapport de l'avant à l'après pour le préambule, de la partie au tout pour la mise en abyme, du visible au lisible pour le prologue.

Si la description est souvent désignée comme un préambule des Géorgiques et comme une anticipation des principaux thèmes de la fiction romanesque et de sa structure, c'est en fonction de sa place dans l'œuvre et de son sujet. Le dessin " préfigure en effet de nombreux éléments du roman à venir, le thème militaire, l'imaginaire de la conquête par la carte géographique, la relation entre deux hommes l'un plus âgé, l'autre plus jeune, "analogue à la relation entre L.S.M. et son descendant" ${ }^{\prime}$ ". Le personnage principal surtout (l'officier vieillissant assis

3. Cora Reitsma-La Brujeere, Passé et présent dans Les Géorgiques de Claude Simon, Amsterdam/Atlanta, Rodopi, 1992, p. 117.

4. "Attaques et stimuli ", entretien entre Claude Simon et Lucien Dällenbach, réalisé le 22 février et le 30 mars 1987, publié dans Lucien Dällenbach, Claude Simon, Paris, Le Seuil, «Les contemporains », 1988, p. 170-181.

5. Nathalie Piégay-Gros, Claude Simon. Les Géorgiques, PUF, «Études littéraires », 1996, p. 20. 
derrière son bureau) y est représenté "avec des détails [...] qui réapparaitront ultérieurement dans les descriptions du général L.S.M. ${ }^{6}$ ".

Le dessin ne se contente pas de représenter par anticipation un personnage: il représente également un objet qui jouera un rôle décisif dans Les Géorgiques. Le moment représenté est « le moment où [l'officier âgé] vient de recevoir un message qui lui a été remis par un officier subalterne encore présent dans la pièce $^{7}$ ». La mise en scène de la transmission d'une lettre sur le dessin anticipe la découverte du secret de famille dissimulé dans un placard par la grand-mère, la missive "concernant l'arrestation du frère Jean-Marie L.S.M., condamné pour émigration et qui sera exécuté en vertu d'une loi que le général lui-même avait fait voter".

Toutefois, si la description du dessin semble préfigurer le roman par la représentation du personnage principal et de la lettre, elle est en réalité, comme l'a montré Brigitte Ferrato-Combe, un "schéma récapitulatif " plutôt qu'un préambule: c'est en effet a posteriori que nous chargeons la description du dessin d'une fonction d'anticipation. En outre, comme l'indique également Brigitte Ferrato-Combe, nous ne reconnaîtrons jamais la scène représentée car elle est absente du roman. Plutôt qu'un préambule, la scène évoquée serait un hors-champ fantasmatique, comme ce secret de famille que le roman découvrira ou comme telle gravure représentant un monstre en frontispice d'une édition du Phèdre de Racine, monstre absent de la pièce, évoqué dans le seul récit de Théramène, mais présent, comme fantasme, dans toute la pièce.

Si nous sommes cependant tentés de retenir le terme de préambule pour cette description, c'est en raison même de sa facture. Il s'agit d'une " esquisse " inspirée, selon Claude Simon, d'un «dessin préparatoire de David pour Le Serment du Jeu de Paume " " et "aussi du portrait inachevé de Bonaparte jeune dont seules la tête et les épaules sont peintes ». En vertu d'une conception téléologique de l'œuvre qui fait du dessin la première étape du tableau, nous sommes conduits à considérer la description comme l'esquisse préparatoire non d'un tableau mais du roman qui va charger et colorer de sang les pâles silhouettes dessinées "à la mine de plomb".

La description du dessin cependant dément son caractère inachevé pour insister, malgré les différences de traitement des parties qui le composent, sur le caractère «fini » de l'ensemble:

Il est significatif que les deux visages ne soient pas seulement esquissés, comme le fait d'habitude un peintre lorsqu'il commence un tableau, posant ici et là quelques rapides

6. Brigitte Ferrato-Combe, Écrire en peintre. Claude Simon et la peinture, op. cit., p. 96.

7. Ibid., p. 97.

8. Ibid., p. 95.

9. "Attaques et stimuli », entretien entre Claude Simon et Lucien Dällenbach, op. cit., p. 170-181. 
touches de couleur pour établir l'harmonie générale, quitte à revenir sur telle ou telle partie, la reprendre même entièrement selon l'évolution de l'œuvre en train: tout au contraire, la facture de chacun d'eux est d'un " fini " poussé jusque dans les moindres détails. ( $G$, p. 15)

La description n'est donc pas une esquisse préparatoire: le " non-fini » fait partie du style de ce dessin qui exhibe ainsi sa facture et nous invite à déplacer notre regard du fait vers le faire (venu du latin facere qui a donné également le " factice », comme le rappelle Claude Simon, qui en fait une caractéristique positive de son « art » dans le Discours de Stockholm ${ }^{10}$ ).

À côté du mot « préambule », le terme de " prologue » est également souvent utilisé pour désigner cette description. Il indique également l'antériorité mais ajoute un logos qui fait du dessin un discours, qui ne retient de l'œuvre plastique que ce qui est soluble dans le texte. La mise en scène d'une lettre est un détail séduisant pour cette lecture attentive à ce que le dessin veut dire. Or la lettre est un trompe-l'œil. Cachée dans le roman, elle est exhibée dans la description du dessin - un peu comme la lettre volée d'Edgar Poe - mais plutôt pour sa dimension visible que pour sa dimension lisible:

On peut voir en effet (quoiqu'elle ait été soigneusement gommée et apparaisse maintenant d'un gris très pâle, comme fantomatique) que la main droite du personnage assis a été primitivement dessinée dans une position différente: détachée de la lettre que continue à tenir l'autre main, elle se soulève un peu, $(G$, p. 16-17)

La présence visible de cette lettre illisible est soulignée, dans la description du dessin, par la mention d'un " repentir ». Ce repentir pourrait être considéré comme un espace transitionnel qui nous fait passer de la description à l'action, c'est-à-dire au récit lui-même, puisque la main a bougé. Une autre lecture est cependant possible: le repentir insiste sur la facture même du dessin, comme Claude Simon lorsqu'il cite ses sources: le dessin décrit est, dit-il, un "dessin imaginaire $^{11}$ " à la manière de David. Rien à reconnaître donc, pas plus les personnages du Serment du Jeu de Paume que le Bonaparte du portrait mais une manière plutôt qu'un sujet, une facture étonnante à laquelle je voudrais m’intéresser à présent.

\section{TRAITS DE STYLE}

La description n'indique pas seulement le sujet représenté mais son traitement, des "anatomies stéréotypées apprises sur l'antique». Le style néoclassique qui, à l'orée du roman, sert à ancrer Les Géorgiques dans une période

10. Claude Simon, Discours de Stockholm, Minuit, 1986, p. 12.

11. "Attaques et stimuli », entretien entre Claude Simon et Lucien Dällenbach, op. cit., p. 170-181. 
historique est caractérisé par des conventions qui étaient déjà raillées par Baudelaire dans l'Exposition universelle de 1855:

Je me rappelle fort distinctement le respect prodigieux qui environnait au temps de notre enfance toutes ces figures, fantastiques sans le vouloir, tous ces spectres académiques; et moi-même je ne pouvais contempler sans une espèce de terreur religieuse tous ces grands flandrins hétéroclites, tous ces beaux hommes minces et solennels ${ }^{12} \ldots$

Il s'agit, pour Baudelaire, de prendre ses distances par rapport à un art du passé opposé à l'art du "peintre de la vie moderne». Ce qui est critiqué en revanche dans la description de Simon est un "code d'écriture admis d'avance par chacune des deux parties, le dessinateur et le spectateur" ( $G$, p. 13). Qu'est-ce donc précisément qu'un "code d'écriture admis d'avance", sinon ce qu'on appelle, en peinture, un académisme? L'observation (le voir) y est remplacée par des conventions de représentation (un savoir) : il s'agit de "signifier " plutôt que de "représenter »:

Ainsi, de même qu'en géométrie descriptive il est convenu que deux droites qui se croisent signifient - et non pas représentent - l'existence d'un plan, l'espace qu'enferment les murs est simplement suggéré, par quelques traits indiquant les arêtes des dièdres ( $G$, p. 13. Je souligne)

Le caractère académique de l'esquisse néo-classique, de ces " anatomies stéréotypées apprises sur l'antique ", de ces " corps figés dans des poses de statues " (que l'on appelle précisément en histoire de l'art des académies) n'est pas seulement soumis à des conventions de représentation, celle des règles abstraites qui fondent «l'illusion perspective » analysée par Erwin Panofsky et que trahit ici l'emploi d'un vocabulaire géométrique ${ }^{13}$. Il est aussi, par le style même du dessin, attentif à des hiérarchies soigneusement détaillées dans la représentation des "frontons" des fenêtres, "triangulaires au premier étage, en arc de cercle au second, les fenêtres du troisième et dernier étage étant entourées d'un simple bandeau en saillie » et dans la différence de traitement des éléments: simple trait, ombres ou couleurs:

Il semble que l'artiste, suivant une sélection personnelle des valeurs, ait cherché, dans la scène proposée, à nettement différencier les divers éléments selon leur importance croissante dans son esprit comme en témoignent les factures particulières dans lesquelles il les a traités, soit, premièrement: les objets inanimés [...] au trait [...]; deuxièmement: la chair, les corps aux muscles, aux veines et aux accidents soi-

12. Charles Baudelaire, exposition universelle, Euvres complètes, éd. Cl. Pichois, t. 2, Paris, Gallimard, «La Pléiade », 1976, p. 583.

13. Erwin Panofsky, Architecture gothique et pensée scolastique, La Perspective comme forme symbolique, Minuit, 1967, p. 39. Cette « illusion perspective » est critiquée également dans La Bataille de Pharsale à propos des Dernières Cartouches d'Alphonse de Neuville, tableau qui « met en scène » la défense de Bazeilles contre les Bavarois en 1870: « Mise en scène toujours mais maintenant ne se contentant plus du simple dièdre formé par la toile de fond et le plancher librement ouvert à droite et à gauche sur les coulisses [...]. Espace clos au contraire décor fermé ", La Bataille de Pharsale, Paris, Minuit, 1985, p. 166. 
gneusement dessinés et ombrés [...]; troisièmement enfin: les têtes des deux personnages qui sont non plus simplement dessinées et ombrées mais peintes à l'aide de couleurs broyées à l'huile, $(G, \mathrm{p} .14$. Je souligne)

Il s'agit d'une mise en ordre du réel analogue à celle qu'effectue L.S.M. dans Les Géorgiques lorsque, élaborant le plan de son domaine organisé en divisions et en sous-divisions, il fait correspondre chaque partie à un code de couleur ( $G$, p. 33). Cette mise en ordre trahit l'illusion d'un pouvoir que le roman va mettre à l'épreuve.

Il s'agit surtout d'une hiérarchie extérieure à l'œuvre plastique qui donne à la tête une supériorité sur la matière corporelle ("la chair, les corps aux muscles, aux veines et aux accidents soigneusement dessinés et ombrés ») et à cette matière humaine la supériorité sur les objets inanimés. Dans le Discours de Stockholm pourtant, Simon réclame, pour le roman comme pour les arts plastiques, "une crédibilité qui soit conférée au texte par la pertinence des rapports entre ses éléments, dont l'ordonnance, la succession et l'agencement ne relèveront plus d'une causalité extérieure au fait littéraire [...] mais d'une causalité intérieure ${ }^{14}$ ».

$\mathrm{La}$ « sélection personnelle des valeurs » établie « selon leur importance croissante dans [l']esprit [de l'auteur] " trahit enfin une perspective intellectuelle, celle idéaliste d'un Vasari lorsqu'il célébrait au XvI ${ }^{\mathrm{e}}$ siècle le dessin comme projet, le disegno: « le dessin n'est rien d'autre que la création d'une forme intuitivement claire et correspondant au concept que l'esprit porte en lui ${ }^{15}$ ".

Contre cette perspective idéaliste, Claude Simon ne cesse de revendiquer un " ordre sensible des choses ${ }^{16}$ ". Le " but de l'art ", indique Claude Simon dans l'un de ses derniers entretiens, est " de donner une sensation de l'objet comme vision et non par connaissance ${ }^{17}$ ". Même affranchie d'une métaphysique, c'est bien en effet l'intervention de l'esprit dans le dessin qui peut en faire une pratique idéaliste. L'esquisse des Géorgiques est à la fois un dessin et un dessein, selon l'ambiguïté orthographique permise en français jusqu'au XviII ${ }^{\mathrm{e}}$ siècle, comme ces " projets " d’architectes, à laquelle elle est précisément comparée: « les objets qui [...] entourent [les corps nus], la pièce où se tiennent les deux personnages, sont figurés avec cette sècheresse qui préside à l'exécution des projets d'architectes » $(G$, p. 12).

14. Claude Simon, Discours de Stockholm, op. cit., p. 22.

15. Erwin Panofsky, Idea. Contribution à l'histoire du concept de l'ancienne théorie de l'art, trad. de l'allemand par Henri Joly [Hambourg, 1924], Paris, Gallimard, 1989, p. 80.

16. Claude Simon, Discours de Stockholm, op. cit., p. 22.

17. Entretien avec Aliette Armel, Le Magazine littéraire, mars 1990, cité par Mireille Calle-Gruber, Claude Simon. Une vie à écrire, Le Seuil, 2011, p. 380. Claude Simon rappelle aussi volontiers cette phrase de Jakobson: "Nous savons le monde, nous ne le voyons pas. ", "Littérature et mémoire ", Quatre conférences, textes établis et annotés par Patrick Longuet, Minuit, 2012, p. 106. 
Lorsqu'il parle de ses œuvres littéraires, Claude Simon indique au contraire combien elles échappent au dessein, au projet initial. Dans le Discours de Stockholm, il cite Valéry qui, affirme qu'il n'a pas "voulu dire mais voulu faire ${ }^{18}$ ". La préface d'Orion aveugle et le Discours de Stockholm disqualifient également la notion de projet, au profit du "bricolage ", du " tâtonnement ", de l'imprévu.

\section{PRATIQUES GRAPHIQUES}

Faut-il donc opposer une théorie idéaliste du dessin à une théorie matérialiste de l'écriture? Pour aborder cette question, il faut d'abord, au-delà des six pages étudiées, élargir notre propos pour y inclure d'autres références à la pratique du dessin dans l'œuvre de Simon.

Constatons, d'abord, que les allusions au dessin y abondent. Constatons, aussi, que ces allusions sont souvent critiques. Dans L'Acacia, le prisonnier échange les pauvres stéréotypes de l'imagerie érotique, "quelque chose qui était au dessin à peu près ce qu'une savonnette est à une pierre ou à une racine", contre des paquets de tabacs "qui constituaient la monnaie du camp ${ }^{19}$ ». Ces dessins nécessitent un laborieux apprentissage:

il répétait les mêmes images du même couple ou de la même femme (il avait appris à leur donner un visage enfantin encadré de chevelures soyeuses) $[\ldots]$ apprenant peu à peu avec une morne perversité à fignoler les détails, les ombres, dessiner les poils avec minutie, faisant cela machinalement, comme il aurait poli des lentilles ou ratissé une cour, se reculant pour apprécier les dégradés et juger de l'ensemble ainsi que peut le faire un émondeur ou un plâtrier ${ }^{20}$,

Ce n'est cependant pas parce qu'ils sont le produit de ce travail «laborieux " qu'ils sont disqualifiés: on sait en effet que les notions de "labeur" et de "travail ", liées à l'artisanat, sont positives pour Simon ${ }^{21}$. Ce n'est pas non plus parce qu'ils représentent des sujets bas, des images pornographiques. Dans La Corde raide (1947), les dessins qu'exécute le narrateur pour Véra la jeune fille russe sont culturellement plus valorisés. Ils sont cependant soumis à la même nécessité fonctionnelle de produire de l'effet sur le destinataire: il s'agit, dans un cas, d'obtenir du tabac, dans l'autre, de séduire une jeune fille:

je lui fis des dessins sur des feuilles de mon carnet. Je dessinai longtemps et un tas de choses. Des cartes d'Europe, des trains, des bateaux, des horloges pour dire l'heure, un cheval parce qu'elle voulait savoir si j'avais été soldat et dans quelle arme, et enfin tous les personnages de la Mythologie grecque, que par chance elle connaissait, pour lui dire qu'elle était belle et que je l'aimais ${ }^{22}$.

18. Claude Simon, Discours de Stockholm, op.cit., p. 23.

19. Claude Simon, L'Acacia, Paris, Minuit, 1989, p. 344 -345.

20. Ibid.

21. Claude Simon, Discours de Stockholm, op. cit., p. 12-13.

22. Claude Simon, La Corde raide, Paris, Le Sagittaire, 1947, p. 15-16. 
Dans ces dessins, il s'agit, comme sur le dessin «à la manière de David " des Géorgiques, d'obéir à " un code d'écriture admis d'avance ", de "signifier " plutôt que de "représenter ». Du client, de la jeune fille à séduire n'est exigée en effet qu'une pauvre reconnaissance, celle du code commun partagé par le dessinateur et le spectateur. Dans La Corde raide, pour Véra, il s'agit explicitement d'un code langagier plutôt que plastique. Représenter des déesses, " pour lui dire qu'elle était belle et que je l'aimais ", c'est remplacer de longs discours par des dessins d'amour.

Ce n'est cependant pas la pratique frustre du dessin d'amateur qui est dévalorisée puisque le dessin d'amateur de l'homme qui renait à la vie à la fin de L'Acacia est, lui, valorisé, précisément parce qu'il annonce l'entrée dans l'écriture:

Un jour il acheta cependant un carton à dessin, du papier, deux pinces et, au cours de ses promenades, il s'asseyait quelque part et entreprenait de dessiner, copier avec le plus d'exactitude possible, les feuilles d'un rameau, un roseau, une touffe d'herbe, ne négligeant aucun détail, aucune nervure, aucune dentelure, aucune strie, aucune cassure ${ }^{23}$.

Ces dessins, comme les dessins du prisonnier, sont le produit d'un laborieux apprentissage. Ce qui les distingue des dessins antérieurs, du dessin des Géorgiques aussi, est moins le sujet que la liberté nouvelle que s'octroie le dessinateur par rapport aux conventions de représentation.

Il renonce, dans ces dessins, à établir une hiérarchie extérieure à l'œuvre, non seulement en représentant, plutôt que des sujets humains, des paysages dont Claude Simon indique combien ils ont eu du mal à s'imposer dans l'histoire de l'art soumise à la hiérarchie classique des genres mais aussi parce que, dans le traitement même du paysage, il privilégie le détail par rapport à la vision d'ensemble. Cette égalité de traitement distingue les œuvres plastiques appréciées par Simon. Lorsqu'il rend hommage à Dubuffet par exemple, Claude Simon souligne l'abaissement de l'homme dans l'œuvre du peintre:

L'une des choses qui me frappe et me séduit (parmi d'autres) c'est dans ces variations, cette partition que vous assignez à l'homme non plus régnant sur mais englobé ou plutôt encastré au sein de la nature (minérale ou végétale) ${ }^{24}$.

Claude Simon, qui ne croit pas plus au progrès dans l'art qu'au progrès dans l'Histoire, construit une généalogie d'artistes qui battent en brèche la hiérarchie anthropocentriste: Breughel, parce que, dans sa peinture, "l'attention est répartie de façon égale sur la totalité de la surface de la toile ${ }^{25}$ ", Dürer, parce

23. Claude Simon, L'Acacia, op. cit., p. 376.

24. Lettre de Claude Simon à Jean Dubuffet du 25 janvier 1983 in Jean Dubuffet et Claude Simon, Correspondance 1970-1984, Paris, L'Échoppe, 1994, p. 57.

25. "Claude Simon: pour en finir avec l'équivoque du réalisme », entretien avec Jean-Paul Goux et Alain Poirson, L'Humanité, 20 mai 1977, p. 8. Repris dans Les Triptyques de Claude Simon ou l'art du montage, Mireille Calle-Gruber (dir.), Paris, Presses de la Sorbonne Nouvelle, 2008, p. 164. 
qu'il donne «la même importance à une hallebarde qu’à un visage humain, à une pierre inerte qu'à un corps en mouvement ${ }^{26}$ ", Rauschenberg qui choisit L'Annonciation de Vinci comme modèle parce que, "sur cette toile, l'arbre, le rocher, la Vierge sont tous d'une égale importance ${ }^{27}$ ". Valorisé dans les arts plastiques, ce mélange des règnes l'est aussi dans l'écriture, celle des Géorgiques notamment, comme l'indique ce passage célèbre où la métamorphose de l'homme en fuite court-circuite toute distinction entre l'homme et l'animal:

(même plus une bête - du moins celles que l'on peut voir courir ou nager: quelque chose comme un de ces organismes à mi-chemin entre le poisson, le reptile et le mammifere qui à l'aube du monde, avant la séparation de la terre et des eaux, se trainnaient dans la vase en s'aidant de choses elles aussi à mi-chemin entre deux noms: déjà plus des nageoires et pas encore des membres) ( $G$, p. 424)

Par sa pratique, Claude Simon dessinateur, attaque également ce qu'on pourrait appeler une conception idéaliste du dessin. Les dessins marginaux, dans les manuscrits, ont surtout été étudiés dans leur relation au texte qu'ils accompagnent ${ }^{28}$. Étudiés pour eux-mêmes, ils proposent parfois d'étonnants contrastes de style comme ces deux dessins de guerrier dans le manuscrit de La Bataille de Pharsale reproduits dans l'ouvrage de Lucien Dällenbach ${ }^{29}$. Le premier, plus élégant, est une reproduction appliquée, à la manière de Polidoro da Caravaggio qui, comme le signale le texte, emprunte lui-même son modèle à Nicolas Poussin. Il cite une tradition idéaliste, une convention de représentation à la manière du dessin d'ouverture des Géorgiques. Le second, plus personnel, s'affranchit, par le réalisme du trait, à la fois de la référence au passé, de la tradition picturale et de l'autorité du grand art. Le dessin "très exact " est, pour Simon, un modèle critique qui impose les lois du « voir » contre celles des savoirs humanistes:

L'humanisme, c'était fini. Sans doute était-ce ce que je ressentais confusément quand je faisais ces dessins très exacts: il n'y a plus de recours, essayons de revenir au primordial, à l'élémentaire, à la matière, aux choses ${ }^{30}$.

On l'aura compris, la pratique du dessin chez Claude Simon trahit une théorie du style et celle-ci concerne également l'écriture. En témoigne un passage non publié sur une page du manuscrit des Géorgiques où l'académisme du

26. Claude Simon, La Bataille de Pharsale, Paris, Minuit, 1969, p. 238.

27. Cité par Lucien Dällenbach, Claude Simon, op. cit., p. 199.

28. On peut citer, par exemple, l'étude de Stéphane Bikialo "Les "tracés sinueux" de Claude Simon: une poétique de la pelote de laine ", Les Images chez Claude Simon, Des mots pour le voir, études réunies et présentées par S. Bikialo et C. Rannoux, La Licorne, $2^{\mathrm{e}}$ semestre 2004, $\mathrm{n}^{\circ} 71$.

29. Lucien Dällenbach, Claude Simon, op. cit., p. 24 et 25.

30. Claude Simon/Marianne Alphant, "Et à quoi bon inventer? ", Libération, 31 août 1989, p. 25. Cité par Didier Alexandre, " "Le fin dessin [...] sur le fond noir". Le récit malgré le monde dans Les Géorgiques", Claude Simon "Les Géorgiques » une forme, un monde, «Lettres modernes », Minard, Caen, 2008, p. 52. 
langage révolutionnaire - «la tacite convention d'un langage maniéré » - est opposé à la "langue sinon neuve, tout au moins tant soit peu en accord avec celle, précise et robuste, de ceux qu'ils se donnaient en exemple ${ }^{31}$ ».

\section{ÉCRIRE CONTRE LE DESSIN?}

Dans la perspective que j'ai esquissée dans cette étude, écrire les Géorgiques serait donc une façon d'écrire contre le dessin d'ouverture, contre ce qui " "signifie[nt]" - et non pas "représent[ent]" ». L'œuvre romanesque y gagnerait la chance d'une étrangeté nouvelle. Ce serait également une façon d'engager le lecteur à découvrir plutôt qu'à reconnaître. 